\title{
When Bees Hamper the Production of Honey: Lexical Interference From Associates in Speech Production
}

\author{
Rasha Abdel Rahman \\ Humboldt-University Berlin
}

\author{
Alissa Melinger \\ Saarland University
}

\begin{abstract}
In this article, the authors explore semantic context effects in speaking. In particular, the authors investigate a marked discrepancy between categorically and associatively induced effects; only categorical relationships have been reported to cause interference in object naming. In Experiments 1 and 2, a variant of the semantic blocking paradigm was used to induce two different types of semantic context effects. Pictures were either named in the context of categorically related objects (e.g., animals: bee, cow, fish) or in the context of associatively related objects from different semantic categories (e.g., apiary: bee, honey, bee keeper). Semantic interference effects were observed in both conditions, relative to an unrelated context. Experiment 3 replicated the classic effects of categorical interference and associative facilitation in a picture-word interference paradigm with the material used in Experiment 2. These findings suggest that associates are active lexical competitors and that the microstructure of lexicalization is highly flexible and adjustable to the semantic context in which the utterance takes place.
\end{abstract}

Keywords: speech production, lexical interference, semantic context effects, semantic associates

A large number of object-naming studies have provided evidence that semantic factors can exert a strong influence on the speed with which we produce language. For instance, in the picture-word interference (PWI) paradigm people display slower naming latency when naming an object while hearing or seeing the name of a semantically related object than when they name an object while hearing or seeing an unrelated name (e.g., Schriefers, Meyer, \& Levelt, 1990). Comparable effects have been observed in the blocking paradigm, in which naming times are contrasted between semantically homogeneous blocks of trials containing objects of a common semantic category (e.g., all are vehicles) and heterogeneous blocks with unrelated objects (e.g., Damian, Vigliocco, \& Levelt, 2001; Kroll \& Stewart, 1994). Paralleling the behaviors elicited within the PWI paradigm, naming is slowed down in homogeneous blocks. This semantic interference effect has considerably shaped our assumptions on how semantic and lexical processing components interact during speech production. It has frequently been interpreted to reflect competition at the level of lexical selection (e.g., Levelt, Roelofs, \& Meyer, 1999; Roelofs, 1992; Starreveld \& La Heij, 1995).

In this article, we explore the nature and scope of semantic interference effects by investigating a striking exception to the

Rasha Abdel Rahman, Department of Psychology, Humboldt-University Berlin, Berlin, Germany; Alissa Melinger, School of Psychology, University of Dundee, Dundee, United Kingdom.

This research was supported by German Research Council Grant AB 277/1-1 to Rasha Abdel Rahman. We thank Gamze Alpay, Friederike Engst, Julia Junker, Kerstin Unger, and David Wisniewski for their assistance in data collection and preparation of the material, and we thank Rainer Kniesche for technical assistance.

Correspondence concerning this article should be addressed to Rasha Abdel Rahman, Department of Psychology, Humboldt-University Berlin, Rudower Chaussee 18, Berlin, Germany 12489. E-mail: rasha.abdel.rahman@cms.hu-berlin.de classic PWI effects described above. Interference from related words has been observed only when these words are members of the target's semantic category (e.g., bee, slug) but not when they are associatively related words from different categories (e.g., bee, honey). This pattern is problematic for models suggesting a lexical competition mechanism because such effects should not be restricted to categorical relations. In these experiments, we used the blocking paradigm to test whether associatively induced lexical interference can be observed and to determine under which conditions the interference can be observed.

\section{Semantic Factors in Models of Speech Production}

The assumption that semantic, syntactic, and phonological processes constitute the basic components necessary for speaking is shared by the majority of theories of speech production (e.g., Caramazza, 1997; Dell \& O’Seaghdha, 1992; Levelt et al., 1999; Starreveld \& La Heij, 1995). However, the models differ considerably with respect to the organization and interactions between these components and with respect to the localization of semantic interference effects. Most important for the present purpose, two types of models can be distinguished, suggesting either lexical (e.g., Bloem \& La Heij, 2003; Bloem, van den Boogaard, \& La Heij, 2004; La Heij, Kuipers, \& Starreveld, 2006; Levelt et al., 1999) or nonlexical (e.g., Costa, Alario, \& Caramazza, 2005; Finkbeiner \& Caramazza, in press; Mahon, Costa, Peterson, Vargas, \& Caramazza, in press) origins of semantic interference effects.

For instance, according to Levelt et al. (1999), object naming as an instance of speech production proceeds as follows. First, the visual presentation of an object activates the concept node (e.g., bee), which then spreads activation to its lexical item, the lemma bee. Simultaneously, activation spreads unconstrained to related concepts that represent the facets of object meaning (e.g., category members such as slug, category descriptions such as animal, and 
associates such as honey), which activate their corresponding lemmas and in turn receive additional activation via feedback from the lemma level. This continuous bidirectional spread of activation-at the conceptual level and between concepts and their respective lemma nodes-results in the mutual activation of semantically interrelated concepts and their lemmas, which then compete for selection.

The competition ends with the retrieval of the lemma with the highest level of activation relative to either one highly active competitor or the sum activation of all its active competitors according to Luce's ratio (cf. Roelofs, 1997). In other words, delays in selection can be caused by either one highly active competitor or by several individually less active lexical competitors (see Roelofs, 1992, for a discussion). The additional presentation of a categorically related word or a categorically homogeneous context increases the amount of competition by directly increasing the activation of one of the competing lemmas and also indirectly strengthening the activation of other related lemmas via shared conceptual features and category nodes. As a result, lemma selection is delayed relative to the presentation of an unrelated word or a semantically heterogeneous context. The proposal for a one-to-many competition mechanism, explicit within this model, is at the heart of the present study and we shall return to it later.

Recently, lexical competition models have been challenged because interference appears to be confined to situations in which the naming response and distractor are at the same level of specificity and in which target and distractor have a categorical relation. For different levels of specificity, the empirical situation is heterogeneous. Some studies report facilitation (e.g., Costa, Mahon, Savova, \& Caramazza, 2003; Vitkovitch \& Tyrrell, 1999), others find interference (Hantsch, Jescheniak, \& Schriefers, 2005), and still others find facilitation for categorization tasks and basic level distractors but find interference for basic level naming and category distractors (Kuipers, La Heij, \& Costa, 2006). In contrast, and most important for the present study, there is strong evidence that the semantic nature of the relation between target and distractor is a critical factor-only categorically, but not associatively, related distractors induce interference. Whereas there are numerous demonstrations of increased naming latencies caused by the presentation of categorically related words, there are literally no reports of similar effects of associatively related words in the PWI paradigm. The latter have been shown either to have no effect on naming latencies or to induce faster naming, particularly when presented prior to picture onset (e.g., Alario, Segui, \& Ferrand, 2000; Bölte, Jorschick, \& Zwitserlood, 2003; La Heij, Dirkx, \& Kramer, 1990; Lupker, 1979) but also when presented simultaneously with the picture. For example, Costa et al. (2005) observed facilitative effects when associates with a part-whole relation to targets were presented simultaneously.

\section{Semantic Activation and the Lexical Status of Associates}

The relation between two concepts at the same level of specificity can be broadly defined in terms of two partially overlapping semantic dimensions: category membership and associative strength (the latter is typically determined by word association norms). Whereas category coordinates have a taxonomic relation that specifies their group membership and shared semantic attributes, associative links are determined by semantic relations irrespective of category boundaries. Two concepts can have a purely categorical relation, a purely associative relation, or a mixed categorical-associative relation. Thus, category members can also be associatively related (e.g., cat and mouse) or not (e.g., cat and snail) and vice versa (for a discussion, see Alario et al., 2000; Lupker, 1985; Perea \& Rosa, 2002). To avoid confounding influences of mixed categorical-associative relations, we defined the two dimensions as being mutually exclusive; category members are items that share a common semantic category node (e.g., animal) and specific features (e.g., has four legs, can move), whereas associates are defined as members of a common semantic frame or context (e.g., apiary: bee, honey, beekeeper) without sharing a category node or a significant amount of semantic features.

To date it is unclear whether the lemmas of semantic associates receive activation during speech planning similarly to semantic category members. Theoretically, given a continuous spread of activation at the conceptual level and assuming that conceptual processing involves the activation of the facets of word meaning, it appears plausible to assume that both categorically related concepts and associatively related concepts from different semantic categories are activated. Furthermore, many models assume that information transmission between concepts and their respective lemma nodes is continuous in nature (e.g., Dell, 1986; Levelt et al., 1999). Thus, if conceptual processing is comprised of associations between target utterances and their various semantic aspects, there is no a priori reason to assume that only category members (and not semantic associates) are active competitors at the lemma level. However, as described above and in clear contrast to these theoretical considerations, there is literally no evidence for competition induced by associatively related words.

Despite the clearly divergent behavioral patterns evoked by these two types of relations, to our knowledge, no production model explicitly incorporates a distinction between category and associative relations. Instead, accounts for the discussed exceptions from classic interference effects suggest semantic, or prelexical, loci. For instance, Kuipers et al. (2006) assumed that semantic facilitation is a consequence of a classic (nonlexical) response congruency effect (Eriksen effect; Eriksen \& Eriksen, 1974), whereas interference reflects lexical competition. If target and distractor converge to the same response at the level of the preverbal message (e.g., a picture of a dog referred to as "animal" and the distractor word "cat"), then naming is facilitated relative to divergent responses (e.g., the distractor word "car").

Other recent proposals have suggested that neither interference nor facilitation effects are located at the lexical level but instead reflect pre- or postlexical mechanisms. Thus, according to Costa et al., 2003, and Costa et al., 2005 (see also Finkbeiner \& Caramazza, in press, for a related postlexical account), semantic interference and facilitation reflect the ease of target concept selection as determined by the combined influences of relatedness and response relevance of the distractor. For instance, mismatching category information of unrelated words can be used to discard these distractors as potential responses earlier than categorically related words, resulting in classic basic-level categorical interference effects. Likewise, associates representing a part of the target do not meet an implicit task criterion of naming whole objects and are therefore dismissed as potential response candidates early in the process, while also priming the target semantic representation 
(Costa et al., 2005). Although these accounts provide straightforward explanations for some effects, their explanatory range is not exhaustive. For example, it is not clear that response congruency underlies facilitated basic-level naming with associatively related target and distractor pairs (e.g., bee and honey), because target and distractor diverge to different responses in a similar way as categorically related pairs. Similarly, it is unclear whether response selection accounts hold for other associative relations for which there is no apparent response criterion allowing for a distinction between target and distractor (for example, bee and honey).

An alternative account that maintains a lexical locus for semantic interference effects can be formulated by capitalizing on the inherent differences in the number of semantic features and category nodes shared by a target object and a categorically related competitor versus an associatively related competitor. When target and distractor are members of the same category, they spread converging activation to a number of competitors that share the category node and several semantic features. As a consequence, all of these active competitors should make their contribution to the delay of target lemma selection, resulting in strong one-to-many competition. In contrast, if target and competitor are associatively related but belong to different semantic categories, activation does not converge onto other related concepts because they do not share a common category node and the number of other shared semantic features is low. Thus, one-to-one competition can be produced but one-to-many cannot.

This difference could explain the asymmetric effects of different types of distractors in analogy to the classic fan effect (Anderson, 1983). This effect refers to the observation of increased retrieval times for a specific piece of information with an increasing number of competing alternatives. That is, the more elements that are connected to a concept, the longer it takes to retrieve any of these elements, with latencies being modulated as a function of the number of alternative connections (the fan). Similar to this fan scenario, an active semantic cohort, consisting of other members sharing common category nodes and/or features ${ }^{1}$ (for instance, the shared category node animal and other common attributes such as legs, fur, etc.) induces strong competition at the lemma level when a category member is presented or when an object is named in a categorically homogeneous block of trials, whereas no such common cohort (or fan) is active when an associate is presented. Therefore, it is conceivable that associates are activated more strongly than unrelated words because of their semantic relation to the target picture but without necessarily inducing strong interference effects. The critical assumption here is that the presentation of associates (as opposed to category members) does not result in the mutual activation of a number of coactivated competing lemma nodes. The observation that associates can induce faster picture naming may reflect relatively strong conceptual links. This, in turn, might enhance target concept and lemma activation more than the competition resulting from a single lexical competitor.

To summarize, we assume that, during picture naming, the concepts and lemmas of associates from different semantic categories are more highly activated than the lemmas of unrelated words without causing the entire activation level of the network to be greatly increased. This account could explain the discrepancy of associate and category member distractor effects without additional assumptions as to why some semantic features of a concept might be activated during picture naming and others might not.
Furthermore, if this assumption is correct, there is no need to dismiss a lexical competition account for semantic interference because of the lack of evidence for the respective effects from associates in the PWI paradigm.

\section{Outline of the Experiments}

We further explored the lexical status of associates by testing for semantic interference effects between associates from different categories with the blocking paradigm. In contrast to the classic PWI procedure, blocking allows for a powerful and flexible manipulation of semantic influences in terms of both categorical and associative relations under comparable conditions, as will be discussed below.

There are two major advantages of the blocking paradigm that should enhance the chances for detecting even small interference effects induced by associates. First, interference can be strengthened and stabilized by repeatedly naming objects in a homogeneous semantic context (cf. Belke, Meyer, \& Damian, 2005). This is presumably because the number of competing items and their activation levels increase with an increasing time interval of coactivation between common category members at the conceptual level and, in turn, at the lemma level of processing. Second, we argue that reciprocal activation of the target and associatively related items can be directly induced by providing a well-defined semantic context in a given block of trials. In other words, by tightly linking associatively related concepts (such as bee, honey, and honeycomb) through a specific semantic context node or semantic frame (e.g., apiary; Fillmore, 1976), we create a situation that is comparable with the interference induced by category members: the mutual, converging activation of a semantic cohort consisting of (associatively) related objects. In contrast to the isolated activation of a single associate in the PWI paradigm, an increasing number of contextually interconnected, and thus coactivated, associate concepts should result in a strong activation of the respective competitors at the lemma level and should therefore noticeably delay target lemma selection.

Further evidence in favor of these predictions comes from recent studies by Damian and Als (2005) and Belke and colleagues (2005), demonstrating that categorical-semantic blocking effects generalize to related, but previously unnamed, objects. This finding suggests that interference effects are at least in part mediated by the activation of a common category node and/or shared semantic features. Consequently, if associates from different categories are coactivated via a common context node, as is the case in the contextually homogeneous blocking condition, the mutual activation of the respective coactivated lemma nodes should result in a classic semantic interference effect. A further prediction of this model, also consistent with Belke et al., is that interference effects should emerge over time but not necessarily in the earliest repetitions, as the convergent activation on the context node and related items needs time to accumulate.

\footnotetext{
${ }^{1}$ Alternative to the activation of a semantic cohort via shared category nodes or features (an assumption derived from the model of Levelt and colleagues, 1999), the presentation of associatively related items could result more directly in the coactivation of the related concepts and lemmas (e.g., Nelson \& McEvoy, 2002). This distinction, however, does not alter our predictions with respect to the categorical and associative effects.
} 
In contrast, models assuming prelexical origins of distractor effects, such as the ease of concept selection or response compatibility, do not anticipate competition effects for associates. Specifically, in the blocking paradigm, there is only one item presented at a time, and naming objects presented in isolation does not seem to fit with the notion of selecting one concept from a potentially response relevant alternative. Thus, although factors such as response relevance or response congruency might play a role in the PWI paradigm, they do not seem to play a major role in the blocking paradigm. Here, one would have to also assume that the objects presented within a given block become relevant responses, which is unlikely as they are not presented together with the target object.

In Experiments 1 and 2, we compared associative and categorical effects using the blocking paradigm. As outlined above, we expected to find interference effects for both associates and category members. In Experiment 3, we further tested our assumption that associative interference can be observed only when the semantic context allows for the converging activation of an associative-semantic cohort. In this experiment, we compared the effects of Experiments 1 and 2 using categorical and associative blocking with the effects induced by the two types of relationships in a classic PWI situation using the same material. According to our assumptions, interference should be observed for category members irrespective of the experimental paradigm and should thus be observed in the blocking situation as well as in the PWI situation. In contrast, associatively induced interference should be observed only in the blocking experiments (Experiments 1 and 2) but not in a classic PWI situation (Experiment 3).

\section{Experiment 1}

In this experiment, we directly compared object naming in heterogeneous blocks of trials with two different semantically homogeneous blocking conditions. The picture stimuli could be presented in blocks consisting of associatively unrelated members of the same semantic category (categorically homogeneous blocks), associatively related objects from different semantic categories (contextually homogeneous blocks), or categorically and associatively unrelated objects (heterogeneous blocks).

For the categorically homogeneous condition, we expected to replicate the classic semantic interference effect that has repeatedly been observed with the semantic blocking paradigm: slower naming latencies in the homogeneous condition than in the heterogeneous condition. If associates are active competitors at the lemma level, then repeated naming in a condition that binds associates in a semantic context should likewise result in semantic interference effects comparable with the well attested categorically induced effects.

\section{Method}

Participants. Fifteen women and 18 men, aged 20 to 32 years ( $M 25$ years), were paid for their participation in the experiment or received partial fulfillment of a curriculum requirement. All participants were native German speakers and reported normal or corrected-to-normal visual acuity and normal color vision. Three participants were excluded because of high error rates.

Material. The target picture set consisted of 25 color photographs of common objects from five semantic categories (animals, food, professions, locations, and tools). The pictures were selected such that they were also orthogonally distributed between five semantic contexts (farm, apiary, sea, forest, and garden; see Appendix A). Thus, within one block of trials, the pictures could either be presented together with other members of the same semantic category (categorically homogeneous condition; e.g.,: cow, bee, fish, deer, slug), together with associatively related members of the same semantic context from different categories (associatively homogeneous condition; e.g., bee, honey, beekeeper, comb, honey extractor), or together with categorically and associatively unrelated pictures (heterogeneous condition). The heterogeneous blocks consisted of five unrelated members from each category and context (e.g., cow, honey, diver, hide, and rake). As for the homogeneous conditions, five different heterogeneous blocks were constructed such that each object was assigned to a specific heterogeneous block. The objects were selected to have minimal visual similarity within the two homogeneous conditions and the heterogeneous conditions and have no phonological overlap. The photographs were scaled to $3.5 \mathrm{~cm} \times 3.5 \mathrm{~cm}$.

Procedure and apparatus. Stimulus presentation on a computer screen and response recording was controlled using Presentation software (Neurobehavioral Systems, Albany, CA). Responses were recorded with a microphone, and naming latencies were measured with a voice key during the entire duration of picture presentation. Naming accuracy and accidental triggering of the voice key were monitored by the experimenter.

Each trial began with a fixation cross displayed in the center of a light grey screen for $0.5 \mathrm{~s}$. Then a picture was presented for $2 \mathrm{~s}$, followed by a blank screen for $1 \mathrm{~s}$. The interstimulus interval was $3.5 \mathrm{~s}$. Participants were instructed to name the picture as fast and as accurately as possible.

Prior to the experiment, participants were familiarized with the pictures and their names in the following manner. All photographs were presented in random order on the screen and participants were asked to name each picture. If necessary, they were corrected or the picture name was provided by the experimenter. After this procedure was repeated twice, participants were given a printed color sheet with all pictures and their names printed below. Then, 15 short experimental blocks of 20 trials each were carried out; the whole session of 300 trials lasted about $30 \mathrm{~min}$. Each picture was presented four times in each condition.

The order of the experimental conditions (categorically homogeneous, contextually homogeneous, and heterogeneous) was counterbalanced between subjects. Furthermore, the order of the different types of blocks within each experimental condition (e.g., animals, food, professions in the categorically homogeneous condition) was also counterbalanced across participants such that every participant had a different order of block types within each experimental condition. Within each block for a given condition and block type, the order of picture presentation was randomized. The four picture repetitions were presented in subsequent blocks, each randomized individually.

\section{Results and Discussion}

Table 1 presents the mean reaction times (RTs) for correct naming trials, standard errors, and the mean percentages of errors in the three experimental conditions separately for the four stimulus repetitions. Figure 1 shows the mean differences between the related and unrelated condition as a function of repetition. Trials with incorrect naming, stuttering, mouth clicks, or vocal hesita- 
tions, and trials with naming latencies deviating more than 2.5 standard deviations from a participant's mean RT in the relevant experimental condition were discarded from the analysis.

Mean RTs were submitted to analyses of variance (ANOVAs) with semantic blocking (categorically homogeneous, associatively homogeneous, or heterogeneous) and repetition (four levels) used as within-subjects factors. All ANOVAs were calculated with participants $\left(F_{1}\right)$ and items $\left(F_{2}\right)$ as random variables. The main effect of semantic blocking failed to reach significance, $F_{1}(2$, 58) $<1 ; F_{2}(2,48)=3.2, M S E=1,467, p=.066, \varepsilon=.71 .^{2}$ However, there was a highly significant effect of repetition, $F_{1}(3$, $87)=79.1, M S E=1,592, p<.001 ; F_{2}(3,72)=80.9, M S E=$ $1,107, p<.001, \varepsilon=.48$, and an interaction between semantic blocking condition and repetition, $F_{1}(6,174)=8.7, M S E=922$, $p<.001 ; F_{2}(6,144)=9.3, M S E=885, p<.001, \varepsilon=.79$.

Numerically, the naming latencies for the first presentation were faster in the two semantically homogeneous conditions than in the heterogeneous condition. This effect was confirmed by post hoc comparisons, yielding significant differences between the heterogeneous and the categorically homogeneous condition, $t_{1}(29)=$ $2.4, p<.05 ; t_{2}(24)=4.1, p<.001$, and between the heterogeneous and the associatively homogeneous condition, $t_{1}(29)=3.5$, $p<.001 ; t_{2}(24)=5.7, p<.001$. In contrast to this facilitative effect, naming was slower in the semantically homogeneous conditions for the fourth presentation for the categorically homogeneous condition, $t_{1}(29)=3.1, p<.004 ; t_{2}(24)=3.2, p<.004$,

Table 1

Mean Naming Latencies (Reaction Times [RTs] in Milliseconds), Standard Errors, and Mean Percentage of Errors (Err) for the Semantic Blocking Conditions in Experiments 1 and 2

\begin{tabular}{cccccccc}
\hline & \multicolumn{3}{c}{ Experiment 1 } & & \multicolumn{3}{c}{ Experiment 2 } \\
\cline { 2 - 4 } \cline { 5 - 7 } Repetition & RT & $S E$ & Err & & RT & $S E$ & Err \\
\hline \multicolumn{7}{c}{ Categorically homogeneous } \\
1 & 673 & 16.0 & 2.8 & 744 & 15.3 & 1.7 \\
2 & 626 & 13.1 & 1.9 & 680 & 12.6 & 1.5 \\
3 & 611 & 12.9 & 1.9 & 683 & 12.2 & 1.7 \\
4 & 623 & 13.8 & 1.7 & 682 & 11.2 & 1.2 \\
5 & & & & 676 & 10.5 & 0.5 \\
6 & & & & 674 & 11.1 & 0.8 \\
\hline
\end{tabular}

Associatively homogeneous

\begin{tabular}{rrrrrrr}
1 & 658 & 14.1 & 1.7 & 752 & 15.9 & 1.2 \\
2 & 611 & 14.8 & 0.4 & 651 & 12.5 & 1.3 \\
3 & 601 & 11.8 & 1.1 & 654 & 14.3 & 0.9 \\
4 & 619 & 12.9 & 1.3 & 650 & 12.0 & 1.2 \\
5 & & & 655 & 13.0 & 0.7 \\
6 & & 645 & 11.6 & 0.5 \\
\hline \multicolumn{7}{c}{ Heterogeneous } \\
1 & 704 & 15.1 & 1.9 & 780 & 14.7 & 2.0 \\
2 & 615 & 12.7 & 1.3 & 650 & 12.5 & 1.5 \\
3 & 601 & 13.3 & 0.9 & 633 & 12.0 & 0.5 \\
4 & 598 & 12.5 & 1.3 & 633 & 12.3 & 0.9 \\
5 & & & 636 & 10.8 & 1.6 \\
6 & & & 634 & 12.6 & .9 \\
\hline
\end{tabular}

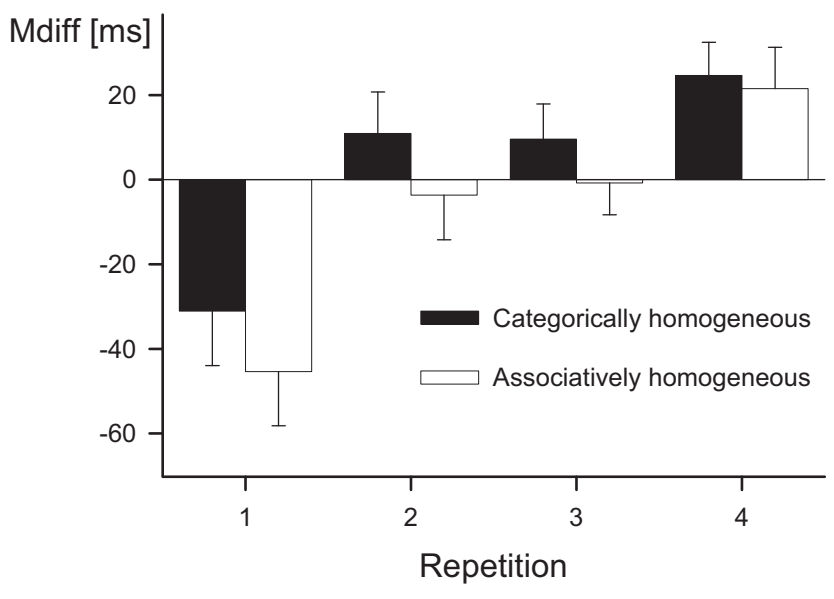

Figure 1. Mean differences between the homogeneous and heterogeneous conditions for each of the four object repetitions in Experiment 1. The error bars depict standard errors.

and for the associatively homogeneous condition, $t_{1}(29)=2.2$, $p<.05 ; t_{2}(24)=3.8, p<.001$ (see Figure 1). In both homogeneous conditions, the latencies for the second and third presentation did not differ significantly from the heterogeneous condition $(t \mathrm{~s}<1.6)$. Thus, the interaction between semantic blocking and repetition can be accounted for by the reversed polarity of the semantic blocking effects in the first repetition in comparison with the polarity of the effects in the fourth repetition and the development of interference effects over time. The error rates did not differ significantly between conditions $(F \mathrm{~s}<1.9)$.

Experiment 1 replicated the categorical semantic blocking effect repeatedly observed by Damian and colleagues (Belke et al., 2005; Damian \& Als, 2005; Damian et al., 2001; Kroll \& Stewart, 1994). The results further indicate that this effect is not necessarily restricted to members of a semantic category but can also be observed for associatively related items (though in smaller numbers). Though the magnitude of the effects was comparatively small in both semantically homogeneous conditions, the pattern of results does not indicate any substantial difference between the respective effects. This finding suggests that associates are indeed activated during speaking at the conceptual level and, as lexical competitors, at the lemma level. An unexpected finding was the observed facilitation induced by both semantically homogeneous conditions compared with the heterogeneous condition for the first stimulus presentation. Before turning to a discussion of the implications, however, we sought to replicate these effects in Experiment 2.

\section{Experiment 2}

The main goal of this experiment was to test whether the rather fragile facilitation and interference effects obtained in Experiment 1 can be replicated and potentially enhanced with new stimulus material and additional stimulus repetitions.

${ }^{2}$ When the sphericity assumption was violated, the respective HuyhnFeldt $\varepsilon$ values for correction of degrees of freedom are reported together with the uncorrected degrees of freedom and the corrected significance levels. 


\section{Method}

Participants. Twenty-one women and 9 men, aged 20 to 37 years ( $M 23$ years), were paid for their participation in the experiment or received partial fulfillment of a curriculum requirement. All were native German speakers and reported normal or corrected-to-normal visual acuity and normal color vision. None of the participants took part in the previous experiment.

Material. A new set of 25 color photographs was composed using the same criteria as in Experiment 1. The objects belonged to five semantic categories (nationalities, headpieces, landscapes, monuments, foods) and were orthogonally distributed between five semantic contexts (China, Saudi Arabia, France, Russia, U.S.A.; see Appendix B).

Procedure and design. Fifteen experimental blocks consisting of 30 trials each were carried out; the whole session of 450 trials lasted about $45 \mathrm{~min}$. Each picture was presented six times in each condition. All other details of the procedure and design were identical to those described in Experiment 1. The same apparatus was used as in the previous experiment.

\section{Results and Discussion}

Table 1 presents the mean RTs for correct naming trials, standard errors, and mean percentages of errors for each of the six stimulus repetitions in the three experimental conditions. Figure 2 shows the mean differences between the related and unrelated conditions as a function of repetition.

An ANOVA using semantic blocking (categorically homogeneous, associatively homogeneous, heterogeneous) and the six repetitions as within-subjects yielded highly significant main effects of semantic blocking, $F_{1}(2,58)=8.7, M S E=4,723, p<.001 ; F_{2}(2,48)=35.3$, $M S E=938, p<.001$, and repetition, $F_{1}(5,145)=156.1, M S E=$ $1,951, p<.001 ; F_{2}(5,120)=118.2, M S E=2,917, p<.001$, and a semantic blocking $\times$ repetition interaction, $F_{1}(10,290)=10.5$, $M S E=1,397, p<.001 ; F_{2}(10,240)=14.6, M S E=819, p<.001$. The significant interaction again reflects a polarity reversal between effects observed in the first repetition and those observed in the remaining repetitions (see Figure 2). For the first presentation, the polarity of the differences reflected facilitation in the categorically homogeneous condition $\left(M_{\text {diff }}=36.2\right), t_{1}(29)=2.2, p<.05$; $t_{2}(24)=3.6, p<.001$. In the associatively homogeneous condition, there was a similar trend toward faster latencies for the first presentation $\left(M_{\text {diff }}=27.0\right)$ in the subjects analysis, $t_{1}(29)=1.7, p=1.0$, which was significant in the items analysis, $t_{2}(24)=3.4, p<.002$. In the subsequent repetitions, the polarity of the effect reverses, revealing interference for both the categorically and associatively homogeneous conditions. Contrasts yielded significant differences between the heterogeneous and the categorically homogeneous conditions for Repetitions $2-6$ ( $t s>2.8, p s<.05)$. In the associatively homogeneous condition, significant interference effects were observed for Repetitions 3-5 ( $t s>2.0, p s<.05)$. The sixth repetition was significant in the items analysis but not in the subjects analysis, $t_{2}(24)=2.3, p<$ .05 , and $t_{1}(29)=1.6, p=1.0$, respectively, and there was no difference between conditions for the second repetition ( $t \mathrm{~s}<0.5$ ).

As Figure 2 shows, the differences between the two homogeneous conditions and the heterogeneous condition remain relatively stable after the first repetition. Indeed, both the main effect of repetition and the interaction vanish in analyses that exclude the first two repetitions (all $F \mathrm{~s}<1.7$, all $p \mathrm{~s}>.1$ ), replicating the pattern observed by Belke et al. (2005). Again, the error rates did not differ significantly between conditions $(F \mathrm{~s}<1.8)$.

In line with our prediction that the mutual activation of related concepts should result in categorically and associatively induced lemma competition, we found robust interference effects for both types of relationship. Furthermore, the interference effects stabilized in the third repetition, which is consistent with our prediction that interference effects (especially those from an induced semantic context binding associatively related items) take time to build up. Overall, this pattern of results confirms our predictions that different types of semantically related items, including associates, are active lexical competitors but that measurable interference effects will be observed only when a significant number of contextually linked coactivated competitors are involved.

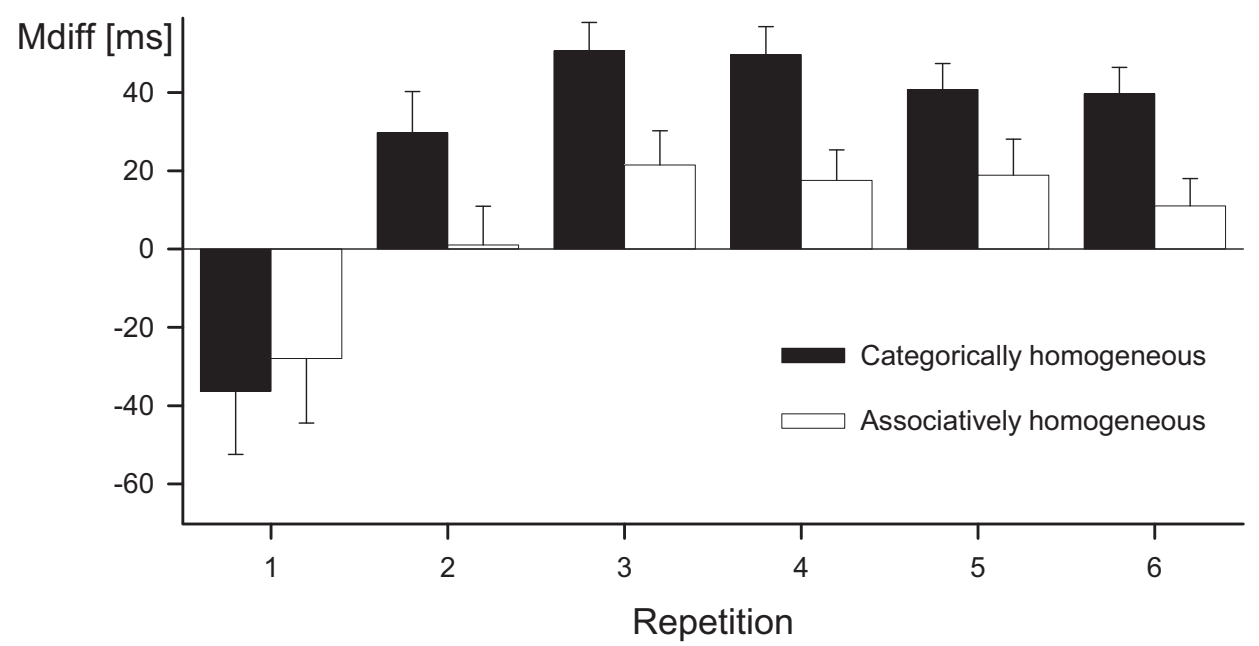

Figure 2. Mean differences between the homogeneous and heterogeneous conditions for each of the six object repetitions in Experiment 2. The error bars depict standard errors. 
However, an alternative account for the findings in Experiments 1 and 2 might be that we happened to construct sets of stimuli in the associative conditions that induce interference irrespective of the activation of a contextually interrelated cohort of competitors. Although this seems unlikely considering that there are no reports of interference from single associate distractors in the PWI literature, it remains a theoretical option to be tested. Therefore, the following experiment was designed to test whether comparable interference can be observed using the same material in a classic PWI situation.

\section{Experiment 3}

The aim of this experiment was to test for categorically and, in particular, associatively induced interference effects from single distractors in a PWI situation with the material used in Experiment 2. If our account of the asymmetrical effects of categorical and associative relations is correct, we should only observe interference from associates in a semantic blocking paradigm (in which the reciprocal activation of an associatively related cohort can be induced by blocking a semantic context) but not in a classic PWI paradigm in which isolated picture and distractor pairs are presented. Thus, presenting the pictures in heterogeneous blocks together with categorically or associatively related distractor words taken from the set of picture names should yield the classic semantic interference effect for category members but not for associates. The latter type of distractors should, as reported in previous studies, yield either no effect or induce facilitation.

\section{Method}

Participants. Nineteen women and 11 men, aged 18 to 29 years ( $M 25$ years), were paid for their participation in the experiment or received partial fulfillment of a curriculum requirement. All participants were native German speakers and reported normal or corrected-to-normal visual acuity and normal color vision. None took part in the previous experiments.

Material. The same set of 25 color photographs from Experiment 2 was used. In addition, the names of the respective pictures were presented auditorily. Each picture was presented together with an auditory word distractor that was (a) categorically related, (b) associatively related, or (c) semantically unrelated (cf. Appendix C).

Procedure and design. Each trial began with a fixation cross displayed in the center of a light gray screen. After $500 \mathrm{~ms}$, the fixation cross was replaced by a picture presented for $2 \mathrm{~s}$, followed by a blank screen for $1 \mathrm{~s}$. A word was presented auditorily to the subject $150 \mathrm{~ms}$ before picture onset. Thus, pictures and distractors were presented with a stimulus onset asynchrony of -150 in all conditions. Participants were instructed to name the objects as fast and as accurately as possible; no instructions were provided regarding the words. The pictures were presented in heterogeneous blocks of trials as described in the previous experiments. Nine experimental blocks consisting of 25 trials each were carried out; the whole session of 225 trials lasted about $20 \mathrm{~min}$. Each picture was presented three times in each condition. All other details of the procedure and design were identical to those of Experiment 1.

\section{Results and Discussion}

Table 2 presents mean RTs for correct naming trials and mean percentages of errors in the three experimental conditions. In com- parison with the unrelated condition, we found slower naming latencies for the categorically related condition $\left(M_{\text {diff }}=14 \mathrm{~ms}\right)$ and faster latencies for the associatively related condition $\left(M_{\mathrm{diff}}=-21 \mathrm{~ms}\right)$. An ANOVA using relatedness (categorically related, associatively related, or unrelated) as a within-subjects factor yielded a significant main effect, $F_{1}(2,58)=18.9, M S E=514, p<.001 ; F_{2}(2,48)=8.4$, $M S E=1,118, p<.001, \varepsilon=.85$. Contrasts between the unrelated and the categorically related conditions revealed significant interference in the subjects analysis, $F_{1}(1,29)=5.9, M S E=992, p<.05$, and a trend in the items analysis, $F_{2}(1,24)=1.8, M S E=2,647, p=.1$. The contrast between the unrelated and the associatively related conditions confirmed the observed numerical facilitation effect, $F_{1}(1$, 29) $=11.8, M S E=1,195, p<.002 ; F_{2}(1,24)=11.4, M S E=1,031$, $p<.002$.

In this experiment, we have replicated the semantic interference effect of categorically related distractor words with the picture-word interference paradigm. However, as predicted, there was no sign of comparable interference from associatively related distractors. Instead, we found robust facilitation induced by the presentation of associatively related distractor words. Thus, the interference observed in the associatively related blocking conditions in Experiments 1 and 2 is not due to the specific material used. The findings of Experiment 3 thus confirm our assumptions on the differential effects of associative and categorical relations during speech planning.

\section{General Discussion}

The current study was designed to explore the nature and extent of semantic activation spreading during speaking. We investigated a marked discrepancy between categorically and associatively induced semantic effects. The question in a nutshell was as follows: Why is it that a categorical relationship reliably induces semantic interference effects, whereas an associative relationship, equally reliably, does not? There are several theoretical options to account for this phenomenon. First, semantic activation during speaking might be restricted to category members and might not involve associative meaning relations. However, to our knowledge, no speech production model makes such a claim, and there is no plausible a priori reason to assume that the activation of the components of object meaning explicitly excludes associative relations.

Alternatively, semantic distractor effects might originate from prelexical rather than lexical factors. As discussed above, such factors could reflect the congruency between the target naming response and the distractor word (Kuipers et al., 2006) or the difficulty of selecting the target semantic representation in the presence of a potentially response relevant distractor word (Costa et al., 2003, 2005). Although these proposals offer an interesting

Table 2

Mean Naming Latencies (Reaction Times [RTs] in Milliseconds), Standard Errors, Mean Percentage of Errors (Err), and Mean Differences Between the Related and Unrelated Conditions in Experiment 3

\begin{tabular}{llllr}
\hline Distractor condition & RT & $S E$ & Err & Difference \\
\hline Categorically related & 854 & 24.2 & 3.3 & 14 \\
Associatively related & 819 & 25.1 & 1.8 & -21 \\
Unrelated & 840 & 23.9 & 2.5 & \\
\hline
\end{tabular}


perspective on possible prelexical mechanisms in the PWI paradigm, they do not seem to offer a straightforward account for semantic effects in the blocking paradigm in which target objects are presented without accompanying distractors. If objects are presented one at a time, as is the case in the blocking paradigm, neither target concept selection nor response congruency should play a major role. Therefore, it seems unlikely that such models can easily provide an explanation for the current findings.

Our proposed account and working hypothesis, derived from the speech production model by Levelt and colleagues (1999), was that associate concepts and their lexical entries are activated during naming. However, interference effects are negligible because of structural characteristics; the presence of associate competitors, as opposed to category members, does not normally result in the mutual activation of a number of coactivated competing lemmas and is therefore not sufficient to result in measurable interference effects. We tested this assumption by creating a condition in which associates are linked by a common semantic context node and thereby, in analogy to a common category node, inducing converging activation from the target object and a number of associates belonging to the context in which the target is named. In short, we matched the conditions under which categorical and associative effects might arise by blocking either a semantic category or a semantic context. This manipulation yielded an effect that has not been observed before; in two experiments with different stimulus materials, we found evidence for both categorically and associatively evoked interference effects. Thus, we concluded that associates actively compete for selection in the course of object naming. This conclusion was further confirmed by a control experiment (Experiment 3) with the PWI paradigm and the same stimulus materials, showing interference effects for categorically related distractors but, most important, no such effects for associatively related distractors. Similar to other studies, we observed faster naming in the presence of associate distractors. Given the findings of all three experiments, it appears likely that interference effects during object naming are not mainly a result of a strong one-to-one competition at the lexical level but rather are primarily determined by the state of activation of all active competitors.

The finding of associatively induced interference is in contrast to the prevailing pattern observed in the PWI paradigm in which, in comparison with unrelated words, associates have either no effect on naming latencies or yield facilitation rather than interference. For instance, Costa et al. (2005) have recently reported robust facilitative effects of associate word distractors on picture naming. In their study, target pictures and associates were presented with a specific type of associative relationship, namely a part-whole relation. This finding questions the lexical competition account for semantic interference, because such effects should not be restricted to a specific type of semantic relation (i.e., category membership). As discussed in the introduction, we entirely agree with the assumption that if interference indeed reflects lexical competition, it should be observed for other semantic relations such as associates. Experiments 1 and 2 show that it can be observed. The critical factor for inducing associative interference was the lexical activation of a common semantic cohort by binding associates through an explicit semantic context that serves, just like shared category nodes, as an interface for the coactivation of the respective items. Furthermore, Experiment 3 demonstrated that the isolated presentation of associate word distractors in the absence of a semantic context is not sufficient to induce interference.

Thus, our account can explain the discrepant effects of different types of semantic relations, as well as the contrasting findings in the PWI and the blocking paradigm, without affording additional assumptions in terms of fundamental differences between categorical and associative activation during speaking and without a need to dismiss a lexical competition account for interference effects. The facilitation observed in the PWI paradigm can be accounted for by assuming that the activation of target concepts by associate distractors is stronger than the competition induced by a single lexical competitor when this competition is not flanked by cohort lemma activation.

Nevertheless, observing interference from associates seems to fly in the face of many years of research using the PWI task. Therefore, it is interesting to note that the overall pattern of results with the blocking paradigm conforms to the more classic picture. Averaging across the four repetitions in Experiment 1, we found numerically slower naming times in the categorically homogeneous blocks than in the heterogeneous blocks, but we found slightly faster naming in the associatively homogeneous blocks $\left(M_{\text {diff }}=29 \mathrm{~ms}\right)$. This overall pattern arises because of the relatively large facilitative effect we found for the first repetition compared with the smaller interference effects observed at the later repetitions. Thus, collapsing across repetitions, the results conform to the pattern previously reported for associates. It is only by looking at each repetition that we can observe evidence for the build-up of converging activation and associative interference via a shared context node.

An alternative interpretation for the observed effects might be that the blocking paradigm does not exhibit lexical competition in a similar way as PWI does but instead reflects other factors such as visual similarity or purely conceptual processing differences. This question has been extensively investigated by Damian et al. (2001), who reported robust interference effects when visual similarity within homogeneous blocks was minimized. Likewise, the stimulus material used in Experiments 1 and 2 of the current study is rather dissimilar, particularly in the critical condition (i.e., the contextually homogeneous blocks). In addition, Damian et al. provided evidence in favor of a lexical rather than a conceptual locus of semantic blocking; interference was not present in a manual (object orientation) classification or in a word naming task. Therefore, we are confident that neither visual similarity nor a purely conceptual locus is a likely alternative explanation for our results. The potential contribution of conceptual factors will be discussed in the following section.

\section{Conceptual Factors and the Semantic Facilitation of Initial Object Naming}

The facilitation observed for the first picture presentation is, in itself, an interesting but unexpected finding. An additional analysis with the two groups of participants in Experiment 1 and 2 revealed this effect to be highly significant in the contextually homogeneous condition in comparison with the heterogeneous condition, $F_{1}(1,58)=12.3, M S E=6,537, p<.001 ; F_{2}(1,48)=41.0, M S E$ $=1,788, p<.001$, as well as in the categorically homogeneous condition in comparison with the heterogeneous condition, $F_{1}(1$, $58)=10.6, M S E=6,411, p<.002 ; F_{2}(1,48)=28.8, M S E$ $=2,323, p<.001$. There were no main effects of group, and there were no interactions between semantic blocking and group $\left(F_{\mathrm{s}}<\right.$ 2). Other studies using the blocking paradigm reported similar 
latencies in the homogeneous and heterogeneous conditions on first naming, but none reported facilitation (e.g., Belke et al., 2005; Damian \& Als, 2005).

One evident design difference to the present study is the inclusion of a practice block before the experiment proper. Here, we did not have such a practice block. Prior to the experiment, all pictures were shown twice on a screen and named in the presence of the experimenter. The familiarity of the stimuli might be a second difference. Some of the depicted objects, particularly objects of the second experiment, were not spontaneously named correctly on first confrontation. For instance, several participants failed to name the Russian chapka or the Chinese bamboo woods when the respective pictures were presented for the first time, whereas naming accuracy was nearly perfect on the second presentation (cf. Table 1). We can only speculate as to why we have found this initial facilitation effect, but judging from the described differences between this and other blocking experiments, it appears likely that the faster naming latencies are a result of facilitated object identification in the semantic conditions and thus are located at the conceptual level.

An alternative explanation might be that participants unsuccessfully attempt to find a common semantic context in the heterogeneous blocks. Because two thirds of the experimental blocks establish a semantic relation between the objects, participants might initially anticipate this to be the case in all blocks. This, in turn, may have resulted in delayed naming latencies in the heterogeneous blocks on first picture naming. ${ }^{3}$ Therefore, participants starting with the semantically homogeneous conditions (two thirds of the participants) should show particularly strong facilitation effects, which are caused by the unsuccessful attempt to create a meaning relation in the heterogeneous blocks. In contrast, participants starting with the heterogeneous condition should not have such a tendency and should therefore not show initial faster naming in the homogeneous conditions in comparison with the heterogeneous conditions.

Alternatively, if the initial facilitation is caused by facilitated object identification in the semantic conditions, participants starting with the homogeneous blocks should benefit from the semantic context supporting the initial identification of individual objects and should not experience much difficulty in identifying the objects in the later heterogeneous blocks. In contrast, participants starting with the heterogeneous condition should have more difficulties with object identification in the heterogeneous blocks than they would in the later homogeneous blocks as there is no supporting semantic context. They should therefore show a stronger semantic facilitation effect. The additional analysis revealed that only participants starting with the heterogeneous condition show semantic facilitation for first picture presentation: $M_{\text {diff }}=77 \mathrm{~ms}$, $F_{1}(1,9)=19.1, M S E=3,124, p<.002$, for the contrast between the categorically homogeneous and heterogeneous conditions in Experiment 1 and $M_{\text {diff }}=116 \mathrm{~ms}, F_{1}(1,9)=42.3, M S E=3,207$, $p<.001$, for the contrast in Experiment 2, and $M_{\mathrm{diff}}=95 \mathrm{~ms}$, $F 1(1,9)=30.5, M S E=2,985, p<.001$, for the associatively homogeneous and heterogeneous conditions in Experiment 1, and $M_{\text {diff }}=106 \mathrm{~ms}, F_{1}(1,9)=42.0, M S E=2,681, p<.001$, for the contrast in Experiment 2. Participants starting with the homogeneous conditions did not show this effect $\left(M_{\text {diffs }}<21 \mathrm{~ms}\right.$, all $F \mathrm{~s}<$ 2.0). Thus, the facilitation on first object naming is most likely due to facilitated object identification rather than the expectation of a meaningful context in the heterogeneous blocks.

\section{Speaking in a Meaningful Context}

The current experiments demonstrate that the context in which we produce language can exert a strong influence on the microstructure of lexicalization. The classic observation that associates have either no effect on naming latencies or induce facilitation (as replicated in Experiment 3) can be turned around into an interference effect by simply providing a semantic context that interrelates associates in a meaningful way (see Experiments 1 and 2). Furthermore, the finding of faster latencies for the first stimulus presentation in Experiment 1 and 2 suggests that naming can be facilitated not only by the presence of associatively related items but also by the presence of categorically related items that typically induce interference rather than facilitation. Thus, we find polarity reversals for classic PWI and semantic blocking effects using the same materials, indicating that the activation of the facets of object meaning during speaking is highly flexible and depends on the context in which the object name is produced. This interpretation confirms recent electrophysiological evidence suggesting that phonological encoding during contextually isolated picture naming can proceed in parallel to the retrieval of semantic object features (Abdel Rahman \& Sommer, 2003; Abdel Rahman, van Turennout, \& Levelt, 2003). In fact, parallel activation of object features and lemmas is a necessary prerequisite for lexical competition in models such as the Weaver ++ model (Levelt et al., 1999) and furthermore prepares the ground for the variable context-dependent semantic effects observed in the current study.

Can this account of our effects shed any light on other polarity reversals observed for categorically related distractor words with mismatching levels of specificity (e.g., Costa et al., 2003; Vitkovitch \& Tyrrell, 1999)? Assuming a flexible architecture of the production system that is shaped by meaningful contexts and task demands, categories might be formed or ignored as the specific context requires. For example, if we were stranded on a desert island, we would quickly create a new and important category of "things used to collect rain water" in a similar way as we induced a category of "things having to do with France" or "things having to do with bees" in the context of associative homogeneous blocks. Likewise, if the task requires speakers to name pictures using more or less specific terms like poodle and lily or animal and plant, the conceptual activation might resonate along more specific or more general conceptual categorical lines such as "type of dog" rather than "type of animal." In turn, basic level distractors might become associate-like in that they specify one aspect of the concept.

Thus, a flexible system as the one sketched here might be able to quickly adapt to the requirements of a specific situation in which an utterance takes place by selectively activating or deactivating contextspecific nodes and the respective items belonging to a given context. Accordingly, we suggest that, similar to the accounts proposed by Costa et al. (2005) and La Heij et al. (2006), some of the observed polarity reversals might be due to adjustments at the conceptual level as to how specific the speaker needs to be or which items are relevant in a given semantic context. What we add to this argument is that this adjustment in turn results in a realignment of salient category nodes through which a cohort of mutually activated lexical entries compete with the target lemma for selection. This context-sensitive alternative

\footnotetext{
${ }^{3}$ We thank a reviewer for pointing this out.
} 
to a hardwired lexicalization system might contribute to our understanding of semantic factors, classic observations, and exceptions in speech production.

To summarize, the common observation that semantic associates do not induce classic interference effects is problematic for models of lexical selection that assume selection by competition (e.g., Dell \& O'Seaghdha, 1991, 1992; Levelt et al., 1999). In this article, we provide evidence that, given a well-defined semantic context, interference from associates can be observed. The critical factor is the converging activation on a shared context node, which functions much as a shared category node does in spreading activation onto a semantic cohort, both at the conceptual and at the lemma level of processing. This evidence provides new insights into the mechanisms underlying lexical selection and might bring us closer to understanding why $d o g$ doesn't always interfere with poodle, whereas bee sometimes interferes with honey.

\section{References}

Abdel Rahman, R., \& Sommer, W. (2003). Does phonological encoding in speech production always follow the retrieval of semantic knowledge? Electrophysiological evidence for parallel processing. Cognitive Brain Research, 16, 372-382.

Abdel Rahman, R., Van Turennout, M., \& Levelt, W. J. M. (2003). Phonological encoding is not contingent on semantic feature retrieval: An electrophysiological study on object naming. Journal of Experimental Psychology: Learning, Memory, and Cognition, 29, 850-860.

Alario, F.-X., Segui, J., \& Ferrand, L. (2000). Semantic and associative priming in picture naming. Quarterly Journal of Experimental Psychology, 53A, 741-764.

Anderson, J. R. (1983, April 1). Retrieval of information from long-term memory. Science, 220, 25-30.

Belke, E., Meyer, A. S., \& Damian, M. F. (2005). Refractory effects in picture naming as assessed in a semantic blocking paradigm. Quarterly Journal of Experimental Psychology, 58A, 667-692.

Bloem, I., \& La Heij, W. (2003). Semantic facilitation and semantic interference in word translation: Implications for models of lexical access in language production. Journal of Memory and Language, 48, 468-488.

Bloem, I., van den Boogaard, S., \& La Heij, W. (2004). Semantic facilitation and semantic interference in language production: Further evidence for the conceptual selection model of lexical access. Journal of Memory and Language, 51, 307-323.

Bölte, J., Jorschick, A., \& Zwitserlood, P. (2003). Reading yellow speeds up naming a picture of a banana: Facilitation and inhibition in pictureword interference. Proceedings of the European Cognitive Science Conference, Germany, 3, 55-60.

Caramazza, A. (1997). How many levels of processing are there in lexical access? Cognitive Neuropsychology, 14, 177-208.

Costa, A., Alario, F.-X., \& Caramazza, A. (2005). On the categorical nature of the semantic interference effect in the picture-word interference paradigm. Psychonomic Bulletin \& Review, 12, 125-131.

Costa, A., Mahon, B., Savova, V., \& Caramazza, A. (2003). Level of categorization effect: A novel effect in the picture-word interference paradigm. Language and Cognitive Processes, 18, 205-233.

Damian, M. F., \& Als, L. C. (2005). Long-lasting semantic context effects in the spoken production of object names. Journal of Experimental Psychology: Learning, Memory, and Cognition, 31, 1372-1384.

Damian, M. F., Vigliocco, G., \& Levelt, W. J. M. (2001). Effects of semantic context in the naming of pictures and words. Cognition, 81, B77-B86.
Dell, G. S. (1986). A spreading-activation theory of retrieval in sentence production. Psychological Review, 93, 283-321.

Dell, G. S., \& O'Seaghdha, P. G. (1991). Mediated and convergent lexical priming in language production: A comment on Levelt et al. (1991). Psychological Review, 98, 604-614.

Dell, G. S., \& O'Seaghdha, P. G. (1992). Stages of lexical access in language production. Cognition, 42, 287-314.

Eriksen, B. A., \& Eriksen, C. W. (1974). Effects of noise letters upon the identification of a target letter in a nonsearch task. Perception \& Psychophysics, 16, 143-149.

Fillmore, C. (1976). Frame semantics and the nature of language. Annals of the New York Academy of Sciences, 280(1), 20-32.

Finkbeiner, M., \& Caramazza, A. (in press). Now you see it, now you don't: On turning semantic interference into facilitation in a Stroop-like task. Cortex.

Hantsch, A., Jescheniak, J. D., \& Schriefers, H. (2005). Semantic competition between hierarchically related words during speech planning. Memory \& Cognition, 33, 984-1000.

Kroll, J. F., \& Stewart, E. (1994). Category interference in translation and picture naming: Evidence for asymmetric connections between bilingual memory representations. Journal of Memory and Language, 33, 149-174.

Kuipers, J.-R., La Heij, W., \& Costa, A. (2006). A further look at semantic context effects in language production: The role of response congruency. Language and Cognitive Processes, 21, 892-919.

La Heij, W., Dirkx, J., \& Kramer, P. (1990). Categorical interference and associative priming in picture naming. British Journal of Psychology, $81,511-525$.

La Heij, W., Kuipers, J.-R., \& Starreveld, P. A. (2006). In defense of the lexical-competition account of picture-word interference: A comment on Finkbeiner and Caramazza (2006). Cortex, 42, 1028-1031.

Levelt, W. J. M., Roelofs, A., \& Meyer, A. S. (1999). A theory of lexical access in speech production. Brain and Behavioral Sciences, 22, 313-335.

Lupker, S. J. (1979). The semantic nature of response competition in the picture-word interference task. Memory \& Cognition, 7, 485-495.

Lupker, S. J. (1985). Relatedness effects in word and picture naming: Parallels, differences and structural implications. In A. W. Ellis (Ed.), Progress in the psychology of language (Vol. 1, pp. 109-142). Hillsdale, NJ: Erlbaum.

Mahon, B., Costa, A., Peterson, R., Vargas, R. A., \& Caramazza, A. (in press). Lexical selection is not competition: A reinterpretation of semantic interference and facilitation effects in the picture-word interference paradigm. Journal of Experimental Psychology: Learning, Memory, and Cognition.

Nelson, D. L., \& McEvoy, C. L. (2002). How can the same type of prior knowledge both help and hinder recall? Journal of Memory and Language, 46, 652-663.

Perea, M., \& Rosa, E. (2002). The effects of associative and semantic priming in the lexical decision task. Psychological Research, 66, 180-194.

Roelofs, A. (1992). A spreading-activation theory of lemma retrieval in speaking. Cognition, 42, 107-142.

Roelofs, A. (1997). The WEAVER model of word-form encoding in speech production. Cognition, 64, 249-284.

Schriefers, H., Meyer, A. S., \& Levelt, W. J. M. (1990). Exploring the time course of lexical access in production: Picture-word interference studies. Journal of Memory and Language, 29, 86-102.

Starreveld, P. A., \& La Heij, W. (1995). Semantic interference, orthographic facilitation, and their interaction in naming tasks. Journal of Experimental Psychology: Learning, Memory, and Cognition, 21, 686-698.

Vitkovitch, M., \& Tyrrell, L. (1999). The effects of distractor words on naming pictures at the subordinate level. Quarterly Journal of Experimental Psychology: Human Experimental Psychology, 52, 905-926. 
Appendix A

Pictures Used in Experiment 1

\begin{tabular}{|c|c|c|c|c|c|}
\hline \multirow[b]{2}{*}{ Contexts } & \multicolumn{5}{|c|}{ Categories } \\
\hline & Animals & Food & Professions & Locations & Tools \\
\hline Apiary & Biene (bee) & Honig (honey) & Imker (beekeeper) & Wabe (comb) & Schleuder (honey extractor) \\
\hline Sea & Fisch (fish) & Garnelen (prawn) & Taucher (diver) & Meer (ocean) & Harpune (harpoon) \\
\hline Forest & Reh (deer) & Braten (roast) & Jäger (hunter) & Hochsitz (hide) & Gewehr (rifle) \\
\hline Garden & Schnecke (slug) & Salat (salad) & Gärtner (gardener) & Beet (garden bed) & Rechen (rake) \\
\hline
\end{tabular}

Appendix B

Pictures Used in Experiment 2

\begin{tabular}{|c|c|c|c|c|c|}
\hline \multirow[b]{2}{*}{ Contexts } & \multicolumn{5}{|c|}{ Categories } \\
\hline & Nationalities & Headpieces & Landscapes & Monuments & Food \\
\hline China & Chinese (Chinese) & Reishut (rice hat) & $\begin{array}{l}\text { Bambuswald } \\
\text { (bamboo woods) }\end{array}$ & $\begin{array}{l}\text { Verbotene Stadt } \\
\text { (Forbidden City) }\end{array}$ & Frühlingsrolle (spring roll) \\
\hline Saudi Arabia & Saudi (Arabian) & Turban (turban) & Oase (oasis) & Mekka (Mecca) & Kichererbsen (chickpeas) \\
\hline France & Franzose (Frenchman) & Baskenmütze (beret) & $\begin{array}{l}\text { Weinberg } \\
\quad \text { (vineyard) }\end{array}$ & Eiffelturm (Eiffel Tower) & Éclair (éclair) \\
\hline Russia & Russe (Russian) & Schapka (chapka) & Tundra (tundra) & Kreml (Kremlin) & Borschtsch (borschtsch) \\
\hline USA & Amerikaner (American) & Cappy (baseball cap) & Prärie (prairie) & $\begin{array}{l}\text { Weißes Haus } \\
\text { (White House) }\end{array}$ & Hamburger (hamburger) \\
\hline
\end{tabular}

Appendix C

Pictures and Distractors Used in Experiment 3

\begin{tabular}{|c|c|c|c|}
\hline \multirow[b]{2}{*}{ Picture name } & \multicolumn{3}{|c|}{ Distractor conditions } \\
\hline & Categorically related & Associatively related & Unrelated \\
\hline Amerikaner (American) & Franzose & Cappy & Oase \\
\hline Cappy (baseball cap) & Turban & Weißes Haus & Eclair \\
\hline Prärie (prairie) & Bambuswald & Amerikaner & Mekka \\
\hline Weißes Haus (White House) & Verbotene Stadt & Hamburger & Saudi \\
\hline Hamburger (hamburger) & Borschtsch & Prärie & Franzose \\
\hline Chinese (Chinese) & Amerikaner & Reishut & Kreml \\
\hline Reishut (rice hat) & Cappy & Verbotene Stadt & Tundra \\
\hline Bambuswald (Bamboo woods) & Oase & Frühlingsrolle & Russe \\
\hline Verbotene Stadt (Forbidden City) & Eiffelturm & Bambuswald & Kichererbsen \\
\hline Frühlingsrolle (spring roll) & Kichererbsen & Chinese & Weißes Haus \\
\hline Franzose (Frenchman) & Saudi & Eclair & Prärie \\
\hline Baskenmütze (beret) & Schapka & Eiffelturm & Frühlingsrolle \\
\hline Weinberg (vineyard) & Tundra & Baskenmütze & Verbotene Stadt \\
\hline Eiffelturm (Eiffel Tower) & Mekka & Weinberg & Schapka \\
\hline Éclair (éclair) & Frühlingsrolle & Franzose & Turban \\
\hline Russe (Russian) & Chinese & Schapka & Eiffelturm \\
\hline Schapka (chapka) & Baskenmütze & Tundra & Hamburger \\
\hline Tundra (tundra) & Prärie & Borschtsch & Chinese \\
\hline Kreml (Kremlin) & Weißes Haus & Russe & Bambuswald \\
\hline Borschtsch (borschtsch) & Eclair & Kreml & Cappy \\
\hline Saudi (Arabian) & Russe & Mekka & Reishut \\
\hline Turban (turban) & Reishut & Kichererbsen & Amerikaner \\
\hline Oase (oasis) & Weinberg & Turban & Borschtsch \\
\hline Mekka (Mecca) & Kreml & Oase & Baskenmütze \\
\hline Kichererbsen (chickpeas) & Hamburger & Saudi & Weinberg \\
\hline
\end{tabular}

Received April 13, 2006 